\title{
'Abd al-Mu'min fils de 'Alī, fils de 'Alwī, fils de Ya'lā al-Kūmī Abū Muhammad
}

L. Golvin

\section{(2) OpenEdition \\ Journals}

Édition électronique

URL : http://journals.openedition.org/encyclopedieberbere/777

DOI : 10.4000/encyclopedieberbere. 777

ISSN : 2262-7197

Éditeur

Peeters Publishers

\section{Édition imprimée}

Date de publication : 1 novembre 1984

Pagination : 64-70

ISBN : 2-85744-201-7

ISSN : 1015-7344

Référence électronique

L. Golvin, «'Abd al-Mu'min fils de 'Alī, fils de 'Alwīi, fils de Ya'lā al-Kūmī Abū Muhammad », Encyclopédie berbère [En ligne], 1 | 1984, document A10, mis en ligne le 01 décembre 2012, consulté le 05 octobre 2020. URL : http://journals.openedition.org/encyclopedieberbere/777 ; DOI : https://doi.org/10.4000/ encyclopedieberbere.777

Ce document a été généré automatiquement le 5 octobre 2020.

(c) Tous droits réservés 


\title{
'Abd al-Mu'min fils de 'Alī, fils de 'Alwī, fils de Ya'lā al-Kūmī Abū Muhammad
}

\author{
L. Golvin
}

1 On ignore la date de la naissance de 'Abd al-Mu'min, mais on sait qu'il vit le jour à Tāğrā, village des environs de Nédroma, en Algérie (ou peut-être nom d'une montagne qui séparait Nédroma de Hunayn), vers la fin du ve/ $/ \mathrm{xl}^{\mathrm{e}}$ siècle. Il appartenait à la famille des Kūmiya (de la confédération des Zanāta). Très tôt, le jeune homme allait révéler d'excellentes dispositions pour les études. De taille moyenne, il est musclé, robuste, son teint est clair et ses traits réguliers. Il s'exprime avec aisance. Son oncle Ya'lū décide alors de l'aider à poursuivre des études dont on ignore la valeur, et il l'accompagnera dans son voyage en direction de l'Orient, en quête de science. Cette expédition ne dépassera pas Bougie où la rencontre avec Ibn Tūmart* décidera de son sort. Il n'est pas sans intérêt de noter que le futur Mahdī des Almohades, qui se pose déjà en réformateur, avait, quelques années auparavant, pris la route de l'orient exactement dans les mêmes dispositions d'esprit que son nouveau disciple. Depuis son départ de sa montagne natale du Haut Atlas, le masmūdien avait beaucoup appris auprès des maîtres à penser de Bagdad, du Caire, peut-être aussi de Damas, et il revenait de son long périple la tête bien pleine sans doute, mais surtout le cœur pur, plein d'un enthousiasme juvénile, décidé à répandre autour de lui la Vérité, à combattre l'erreur et à imposer à ses coréligionnaires les principes d'un islam rénové, scrupuleusement attaché à l'unicité d'Allah. Son voyage de retour s'effectuait en étapes plus ou moins longues, jalonnées d'exploits retentissants assez significatifs de ses futures intentions. A Bougie, en milieu berbère toujours attentif aux échos venus d'Orient, toujours prêt à y trouver des éléments de querelle susceptibles de secouer le joug du pouvoir établi, le « Faqih du Sus » trouvait, à n'en pas douter, un écho favorable et il est probable que ses paroles éveillaient des sentiments qui ne demandaient qu'à s'exprimer. Pourtant, la rudesse du réformateur trop zélé qui s'exprimait dans la cité par des actions brutales, lui avait valu quelques ennuis et avait amené les dirigeants ḥammādides à l'expulser, l'obligeant à se réfugier à Mallala, un faubourg, où il poursuivait dans une mosquée sa 
mission de propagande. C'est là que, en 511/1117, il devait recevoir le jeune 'Abd alMu'min. Les deux personnages étaient faits pour s'entendre. On peut penser que, d'emblée, le neveu de Ya'lū fut conquis et littéralement subjugué. Il résolut alors de se joindre au petit groupe d'adeptes, presque tous contribules du Maitre, et de s'attacher, comme eux, aux pas du futur Mahdī.

Nedroma. 'Abd el Mumin naquit à Tagra, village proche de Nedroma (Photo G. Camps).

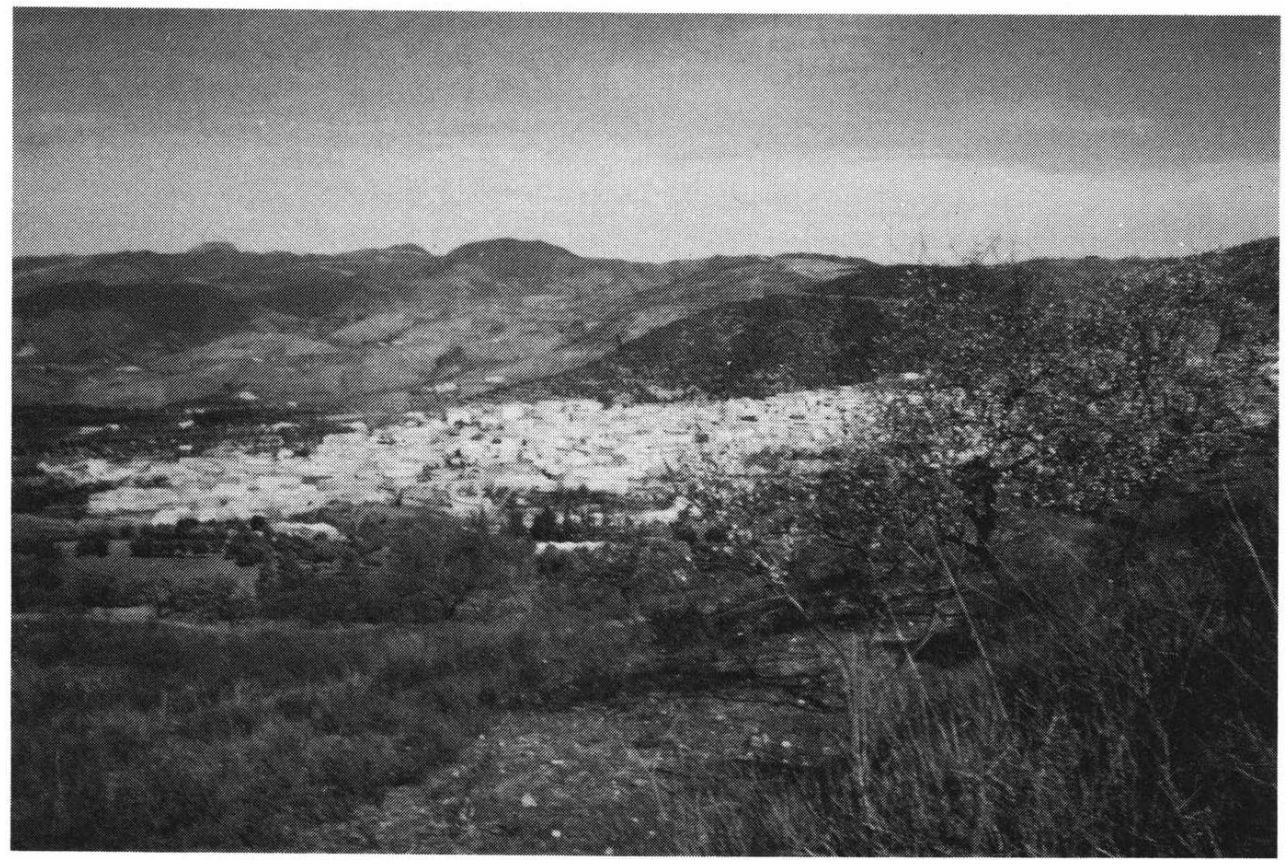

2 On connaît la suite de ce voyage marqué par de nombreux incidents de rue provoqués par la farouche intransigeance de Ibn Tūmart. Tour à tour, Tlemcen, Nédroma, Taza, Fès, Meknès, Marrakech enfin, furent le théâtre de ses sermons et de ses exploits spectaculaires assortis d'expulsions la plupart du temps. Pourtant, en dépit de ces incidents, sans doute consciemment provoqués, la doctrine de l'unitarisme faisait son chemin et gagnait à sa cause de nombreux adeptes. Partout le grain était semé qui ne tarderait pas à germer et à lever.

Ibn Tūmart avait jugé d'emblée les qualités de son disciple et il lui avait accordé très tôt un ordre de préséance à côté des grands chefs masmūdiens ; mieux encore, on peut dire qu'il l'adoptera en réussissant à l'intégrer à sa propre tribu, celle des Harǵa où il siégera au conseil des dix. 'Abd al-Mu'min devient alors le plus brillant propagandiste du Maître, il sera de toutes les expéditions et révèlera ses qualités de guerrier. Pourtant, à la mort du Mahdī (ramaḍān 524/septembre 1130), il faudra toute l'insistance de Abū Ḥafș 'Umar İntī* pour qu'il soit reconnu successeur du Maître. Comme il arrive dans le monde musulman, la mort du chef a été tenue secrète afin de permettre au conseil des dix de s'accorder sur le choix du successeur ; on devine que la fameuse "Loi du sang ", lien quasi organique des tribus, ne pouvait accepter sans contestation l'étranger, le Zanata venu du Maġrib central. Trois ans s'écoulèrent ainsi. Enfin, au retour d'une expédition victorieuse contre les Gazzūla, on se résolut à annoncer le décès du Mahdī et à proclamer 'Abd al-Mu'min, décision qui allait assurer le triomphe du mouvement. Jusque là, en effet, les succès des unitaristes n'avaient pas dépassé le cadre local et, la seule expédition en direction de Marrakech s'était soldée par un échec. 
Les conquêtes de 'Abd al Mu'min.

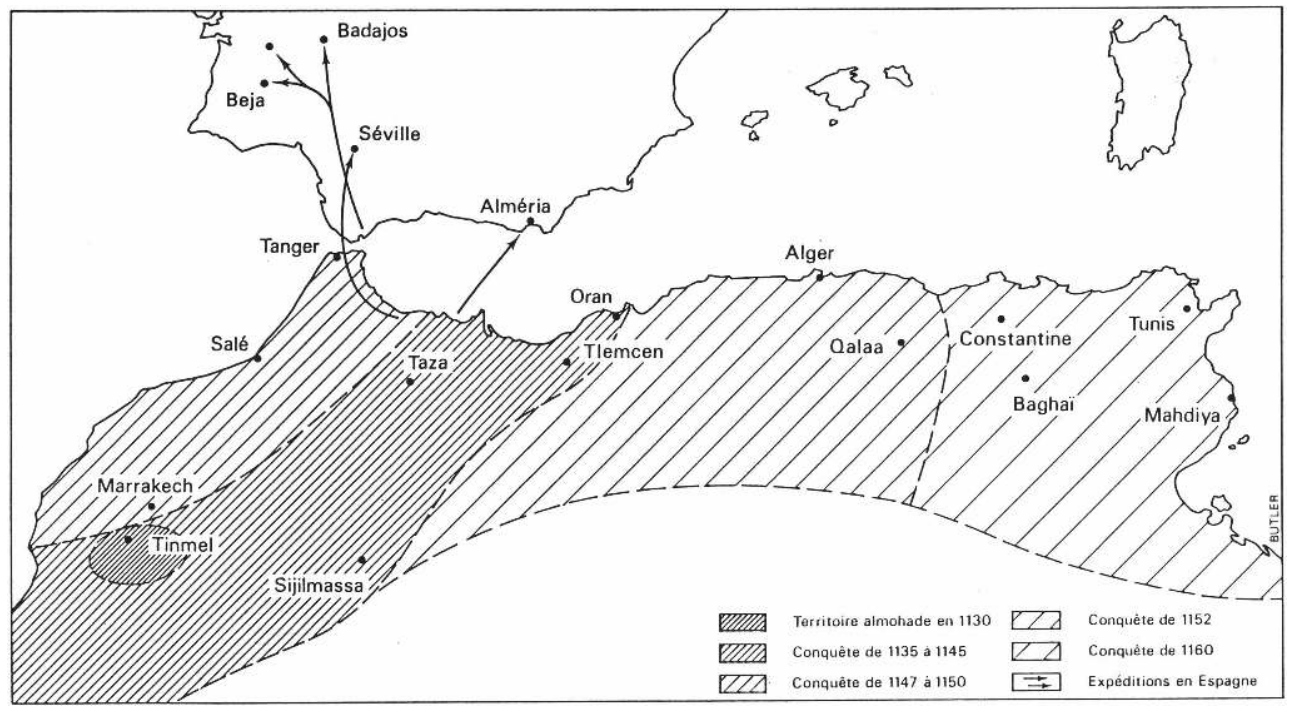

4 Il n'est pas trop tôt de dire que l'immense succès des Masmūda fut certes dû aux qualités guerrières de l'homme; intelligent, éloquent, il avait à n'en pas douter le prestige du chef, mais son ascendant naturel en imposait d'autant plus aux Berbères du Haut Atlas qu'il était étranger aux clans, de tous temps hostiles les uns aux autres, comme il est fréquent dans une telle société.

Trop habile pour renouveler l'erreur de descendre dans la plaine et de se mesurer aux troupes almoravides de Marrakech, le nouveau maître des Almohades commença par s'assurer quelques positions fortes en bordure de la montagne, puis il s'enhardit dans le Sūs et dans le Dar'a ralliant de nombreuses tribus. De 535/1141 à 539/1145 il lance une grande offensive sur le Moyen Atlas et s'empare de Taza, clef du Magirib central, puis du Rif, et il atteint la côte méditerranéenne. Il étend alors ses conquêtes vers l'Est et s'empare de Nédroma, siège de sa tribu d'origine. Tlemcen assiégée devait se rendre assez rapidement tandis que le chef berbère était déjà aux portes de Fès. L'ancienne capitale résista neuf mois puis succomba à son tour (540/1146). 'Abd al-Mu'min était alors maître de tout le Nord du Maroc et il tenait solidement la montagne berbère. Le moment était venu de donner le coup de grâce à la dynastie almoravide. Marrakech devait résister héroïquement dans sa Qasba, mais elle fut contrainte de capituler en šawwal 541/avril 1147. La famille régnante fut sauvagement exterminée. Le vainqueur s'installa dans le propre palais des Almoravides, mais il n'allait pas tarder à faire édifier dans la capitale du nouvel empire la grande mosquée dite Kutubiyya. Déjà, il avait affirmé ses ambitions de constructeur à Taza, et Tinmāl voyait le modeste oratoire d'Ibn Tūmart faire place à une très belle mosquée. Dans ces réalisations architecturales s'exprimait une très nette évolution de l'art musulman d'Occident qui atteignait alors sa pleine maturité.

Mais, sur le plan politique, il restait à parfaire la conquête du Maroc en pacifiant la plaine atlantique, ce qui devait demander quelques années d'efforts. Après quoi, 'Abd al-Mu'min entreprenait un tri rigoureux de ses fidèles. La purge atteignit quelques milliers de victimes soupçonnées, à tort ou à raison, de menacer la nouvelle puissance berbère. 
7 La solidité des arrières assurée, le chef des Almohades résolut de conquérir tout le Maǵrib. Il se prépara soigneusement pendant plusieurs années à cette grande expédition. La situation du pays ne manquait pas d'être préoccupante. En Ifrīqiya, les Zīrides étaient aux abois. Défendant à grand peine le littoral contre les entreprises de Roger II, roi normand de Sicile. L'intérieur du pays leur échappait, envahi par les Arabes nomades hilāliens. Seule Tunis voyait sa population s'accroître et la ville connaissait une certaine prospérité sous les B. Hurasān. Au Maġrib central, les Hammādites avaient dû abandonner en partie leur capitale de la Qa'la où ne résidait plus qu'une modeste garnison sous les ordres d'un des fils du prince régnant installé à Bougie : Yahyā fils de 'Abd al-'Azīz. La nouvelle capitale, bien protégée par l'écran de la montagne kabyle se développait harmonieusement et les Hammādides tenaient la côte jusqu'à Alger, mais ils ne pouvaient s'aventurer vers l'intérieur où s'étaient infiltrés les Hilāliens qui maintenaient le pays en pleine anarchie.

La mosquée de Tinmāl, plan d'après $H$. Terrasse.

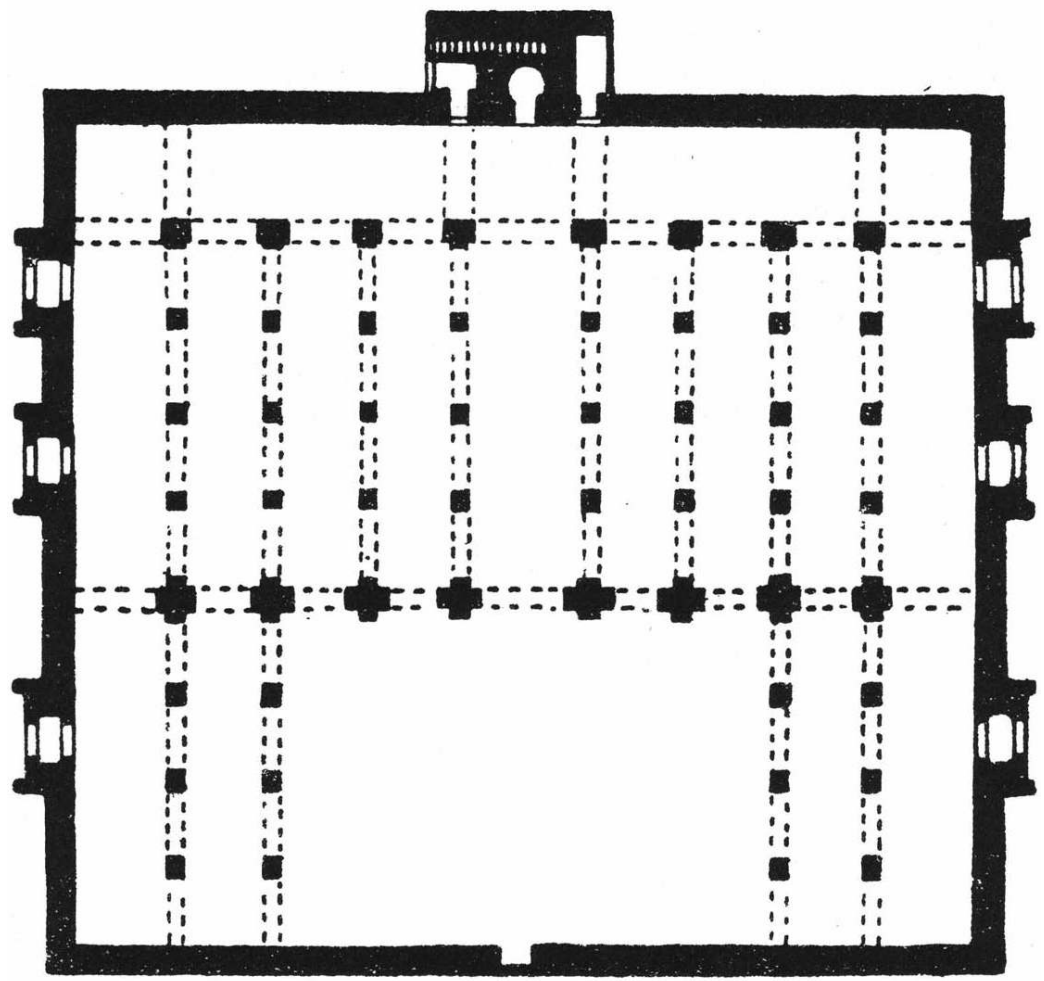

8 Ayant concentré ses troupes à Salé où elles avaient été fort bien entraînées au combat, le chef almohade se décida à déclencher l'offensive qui devait se solder par une série de victoires : Alger, Bougie, Sétif, la Qa'la, n’offrirent que peu de résistance. En 547/1152, le royaume des Ḥammādides avait vécu. Les Arabes de la plaine, impressionnés, se ralliaient, 'Abd al-Mu'min exerçant à leur égard une politique d'attirance, sans doute dictée davantage par la prudence que par la conviction. Cette prudence, une des qualités essentielles de 'Abd al Mu'min, devait lui faire remettre à plus tard la dernière entreprise : la conquête de l'Ifrīqiya. Pendant huit années il prépara cette expédition à Salé, levant une armée de 200000 hommes, préparant soigneusement l'intendance, ne laissant rien au hasard. En 554/1159, il se mit en marche, divisant l'expédition en plusieurs corps selon des itinéraires différents. Tunis atteinte se rendit après un court siège, Mahdiya, aux mains de Roger II, opposa une plus sérieuse résistance, mais la ville 
dut capituler en muharram 555/janvier 1160. Toutes les autres cités de l'ancien royaume des Zïrides se rendirent. Pour la première fois de l'histoire, tout le Maǵrib se trouvait sous les ordres d'un seul chef.

Mais, depuis longtemps déjà et pendant même ces expéditions, 'Abd al-Mu'min avait les yeux fixés sur l'Espagne. Dès 539/1145 le pays entrait en rébellion contre ses gouvernants almoravides et retournait à l'anarchie politique des Mulūk al-Ṭawā'if si propre à favoriser les entreprises de reconquête chrétienne. Seules les Baléares restaient sous l'autorité almoravide en la personne des Banū Ǵaniya qui n'allaient pas tarder à se manifester au Maǵrib et à causer bien des soucis aux Almohades et, en Ifrīqiya, à leurs successeurs les Ḥafșides.

Dès 541/1147, 'Abd al-Mu'min s'était décidé à intervenir en envoyant un corps d'armée en Andalousie. Les Almoravides furent pourchassés, se repliant vers la côte et abandonnant Séville. En 551/1157 un nouveau corps de troupe avait repris Alméria tombée entre les mains des Chrétiens. Cependant, le chef almohade était trop occupé en Afrique du Nord pour s'imposer comme il le désirait. En 1160, les mains libres, il se tourna sérieusement vers l'Espagne et, passant le détroit, il établit son camp à Gibraltar qu'il fit fortifier. Les Chrétiens furent vaincus à Badajoz, à Béja et à Evora, puis à Carmona. Mais se n'était là que parer au plus pressé. Rentré à Marrakech au début de 558/1162, le Sultan fit concentrer une armée, selon son habitude, à Salé, au Ribāt alFatḥ (future Rabat) où il se rendit en personne, préparant, avec sa minutie habituelle, une vaste expédition, mais la mort devait le surprendre en jumādā II 558/mai 1163 ; il fut inhumé à Tinmāl au côté de son maître Ibn Tūmart, à proximité de la mosquée qu'il avait lui-même fait construire.

11 Dès la prise de Marrakech qui assurait son triomphe sur les Almoravides, 'Abd alMu'min avait pris le titre califien de Amīr al-Mu'minīn, marquant ainsi sa volonté de rompre avec le califat 'abbāside d'Orient paralysé par les Turcs et affirmant également ses prétentions de réformateur de l'Islam, du moins dans tout l'Occident où il entendait imposer la doctrine définie par son maître le Mahdī impeccable Ibn Tūmart.

Chef berbère (bien qu'il ait revendiqué une ascendance arabe et qu'il se soit fait établir, comme il se doit, une généalogie le rattachant à l'illustre famille du Prophète) 'Abd alMu'min devait organiser l'administration de son empire selon des principes essentiellement berbères adoptés par Ibn Tūmart. L'organisation tribale traditionnelle changeait de taille, elle tentait de s'adapter à l'échelle du Maǵrib. Les Berbères triomphants devenaient réellement maîtres de l'Afrique du Nord, des côtes atlantiques aux Syrtes. Arabes des villes ou arabes nomades des campagnes durent se soumettre à un système où ces derniers pouvaient d'ailleurs retrouver des coutumes familières à tous les nomades. Une hiérarchie rigoureuse s'établit, dominée par les tribus du Haut Atlas marocain, alliés de la première heure (il est à noter que 'Abd al-Mu'min avait réussi à faire admettre sur le même pied ses contribules, les Kūmiya); venaient au second rang les ralliés de la seconde vague à l'exclusion de certaines tribus. Tous eurent leurs délégués, les ŠayH-s, auxquels le Sultan-Calife accordait la plus grande attention. Le plus noble d'entre eux Abū Ḥafș 'Umar, compagnon de Ibn Tūmart, apparaissait comme une sorte de premier ministre, mais il devait partager son autorité avec le "Conseil des Dix ». Il est évident néanmoins que l'autorité suprême restait entre les mains du chef qui était le grand maitre de l'appareil, apanageant ses propres fils tout en leur adjoignant des ŠayH̆-s masmūdiens. Dès 549/1154, rompant en cela avec la vieille tradition tribale, il avait réussi habilement à faire désigner comme successeur 
son fils Muhammad, mais, lorsqu'il se ravisera quelques années plus tard pour tenter de faire reconnaître de préférence un autre fils, Yūsuf, il rencontrera l'opposition de Abū Hafș ‘Umar qui ne se ralliera définitivement que quelques années après la mort de 'Abd al-Mu'min.

\section{BIBLIOGRAPHIE}

Anonyme. Kitāb al-Ansāb (recueil factice de la bibliothèque de l'Escorial) al-Baydaq (Abū Bakr b. 'Alī al-Ṣanhāḡīi) v. Lévi-Provençal (E.), Documents inédits d'histoire almohade. Paris, 1928-Ibn alQațtān.

Nazm al-Ǧumān, v. Lévi-Provençal, Six fragments inédits d'une chronique du début des Almohades, Mélanges René Basset, Paris, 1925, II, p. 335-393.

LEVI-PROVENÇAL E. Trente-sept lettres officielles almohades. Rabat, 1941.

LEVI-PROVENÇAL E. Un recueil de lettres officielles almohades. Paris, 1962.

AL-MARRAKUŠI ‘ABD AL-WĀḤID. Mu’ğib, éd. Dozy.

IBN ABĪ ZAR`, Raw ḍ al-Qirțās, éd. Tornberg, 2 vol. , Upsala, 1843-46, trad. Beau-mier, Paris, 1860.

Al-Hulal al-Mawšiyya, éd. I.S. Allouche, Rabat, 1936, trad. espagnole de A. Huici Miranda, Tetouan, 1952.

IBN AL-AṬīR. Kāmil fì l-Ta'riH, éd. C. J. Tornberg, 13 vol. , Leide, 1867-74, trad.

Fagnan, Annales du Maghreb et de l'Espagne, Alger, 1901.

IBN AL-HูĀṬIB. A'māl al-a'lām.

IBN HALDŪN. Histoire des Berbères, texte I, trad. II.

FAGNAN E. Histoire des Almohades d"Abd al-Wâhid Merrâkechi. Alger 1893.

ZARKAŠI. Ta'riH al-Dawlatayn. Tunis, 1289, nelle éd., Tunis, 1966.

IBN HुALLIKĀN. Wafayāt al-A'yān. I. p. 390-391.

IBN ‘IDĀRI. Al-Bayān al-Mugrib. Dozy, Leyde, 1848, éd. Huici Miranda et trad, espagnole, Tétouan, 1953-1954.

MARÇAIS G. La Berbérie musulmane et l'Orient au Moyen-Age. Paris, 1946.

MILLET R. Les Almohades. Paris, 1923.

TERRASSE H. Histoire du Maroc. Casablanca, 1949, p. 261-367.

MERAD A. 'Abd al-Mu'min à la conquête de l'Afrique du Nord Contribution à l'histoire de la dynastie mu'minide. In Annales de l'Inst. d'Et. Orientales, t. XV, 1957, p. 109-163 et t. XX, 1962, p. 401-478.

DEVERDUN G. Marrakech, des origines à 1912. 1959, p. 151-301.

LE TOURNEAU R. The Almohad Movement in North Africa. Princeton, 1968. 
INDEX

Mots-clés : Biographie, Espagne, Maroc, Moyen Âge 